\title{
Superluminal Tunneling of a Relativistic Half-Integer Spin Particle through a Potential Barrier
}

\author{
Luca Nanni \\ PhD Independent Scholar, University of Ferrara, 44121 Ferrara, Italy \\ luca.nanni@student.unife.it
}

\begin{abstract}
This paper investigates the problem of a relativistic Dirac half-integer spin free particle tunneling through a rectangular quantum-mechanical barrier. If the energy difference between the barrier and the particle is positive, and the barrier width is large enough, there is proof that the tunneling is always superluminal. For antiparticle states, the tunneling may be either subluminal or superluminal instead, depending on the barrier width. These results derive from studying the tunneling time in terms of phase time. For particle states these are always negatives while for antiparticle states they are always positives, whatever the height and width of the barrier. The scattering also leads to an anomalous distortion of the Dirac spinor that tends to disappear as the particle velocity approaches the speed of light. Moreover, the phase time tends to zero, increasing the potential barrier both for particle and antiparticle states. This agrees with the interpretation of quantum tunneling that the Heisenberg uncertainty principle provides. This study's results are innovative with respect to those available in the literature. Moreover, they show that the superluminal behaviour of particles occurs in those processes with high-energy confinement.
\end{abstract}

Keywords: quantum tunneling; evanescent waves; Hartman effect; relativistic particles

PACS: 03.65P

\section{Introduction}

Several theoretical and experimental studies in the past decades have examined phenomena involving superluminal waves and objects because of their implication in quantum and cosmological physics [1-6]. Among them, the study of the tunneling time problem is one of the topics that has most attracted the interest of quantum physicists [7-12]. Researchers have approached this issue both from the perspective of non-relativistic [13] and relativistic [14] quantum theory. In both cases the tunneling time does not depend on the barrier width (at least for large enough barriers), thus proving superluminal behaviour of the quantum object (wave or particle). However, the tunneling time problem remains a controversial one in quantum physics. A comprehensive and clear theory to explain how long does it take a particle to tunnel through a barrier still does not exist [15]. As is well known, classical quantum mechanics does not treat time as an Hermitian operator but rather as a parameter [16]. Time does not appear in the commutation relationships typical of the Hermitian operators, even if it appears in one of the forms of the Heisenberg uncertainty principle, being a physical variable conjugated to the energy. For this reason we have to give up directly knowing the tunneling time. We may bypass the obstacle by assuming that the wave packet inside the barrier is stationary, with an imaginary wave vector. We can then interpret the tunneling time as the phase variation of the evanescent stationary wave that crossing 
the potential barrier induces. This is the definition of tunneling phase time [17-18] and is the most widely used quantity in the studies of tunneling phenomena.

In this paper we investigate, by the study of the phase time, the one-dimensional scattering process of a relativistic half-integer spin free particle through a rectangular barrier. When the barrier height is greater than the particle energy, for particle states we prove that the tunneling process always occurs at superluminal velocity. This transpires regardless of the width of the barrier and the energy gap between the barrier and the relativistic particle. Antiparticle states, on the contrary, behave in a different way, and the scattering through the barrier may be subluminal or superluminal, depending on the barrier width. Furthermore, the study shows that when the energy gap increases, the tunneling time tends to zero for both particle and antiparticle states, and the crossing velocity of the barrier diverges to infinite values. We also investigate the phase time delay of the second spinor component, proving that a spinor distortion occurs when the particle velocity is quite far from its relativistic behaviour. This peculiarity disappears as the particle velocity increases, i.e., when the second spin component becomes more and more important (its magnitude increases as the relativistic factor $\beta=u / c$ increases).

\section{Quantum Tunneling from the Perspective of the Heisenberg Uncertainty Principle}

In 1928 George Gamow gave a simple but elegant interpretation of the quantum tunneling [18]: the particle with energy $E$ borrows from the vacuum an amount of energy $(U-E)$ to surmount the barrier potential $U>E$. Recalling the uncertainty principle in the form:

$$
\partial E \partial t \geq \hbar
$$

provides the time needed to repay the borrowed energy as:

$$
\partial t=\frac{\hbar}{(U-E)}
$$

Eq. (2) gives the tunneling time, i.e., the time the quantum particle needs to cross the potential barrier. It is interesting to note that this time does not depend on the geometry of the barrier, providing simple evidence of the Hartman effect [11]. If $a$ is the one-dimensional barrier width, the tunneling velocity is:

$$
v_{t}=\frac{a}{\partial t}=\frac{a(U-E)}{\hbar}
$$

Since the barrier width and the energy gap can assume whatever value, from Eq. (3) we conclude that there are geometrical and energetic conditions under which the tunneling is a superluminal process. In particular, for large values of $a$ (high confinement) and $(U-E)$ (highenergy systems) the tunneling velocity inside the barrier is higher than the speed of light, according to a previous work by the present author [20].

\section{The Scattering Model}

Let us consider a half-integer spin relativistic free particle of rest mass $m_{0}$, energy $\pm E$ (depending on its particle or antiparticle state) and velocity $u$, scattering through a potential barrier of width $a$ and potential energy $U$. Theoretically, the potential energy is greater than the particle energy $(U> \pm E)$ : 


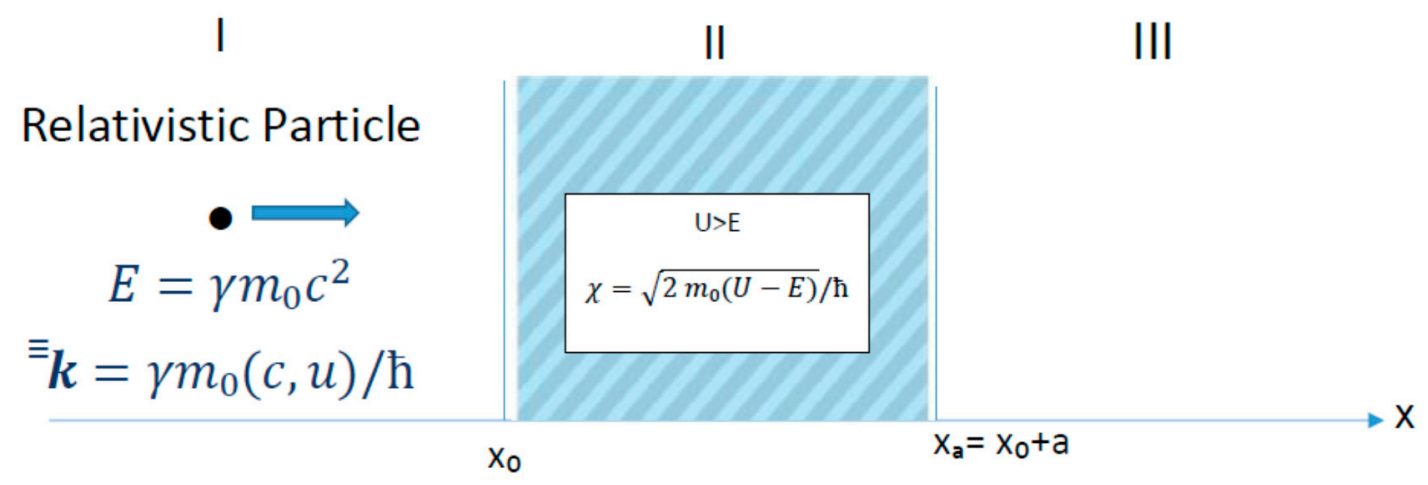

Figure 1: Particle scattering through a potential barrier: in regions I and III the particle behaves as a free relativistic quantum object. In region II the particle behaves in a transcendent way.

In regions I and II, where the potential energy is zero, the particle moves freely and behaves like a wave-packet [21], which spreads in space-time. To simplify the study we suppose a twodimensional space-time, so that the plane wavefront impinging on the barrier has the twocomponent spinor form (there is no spin-flip):

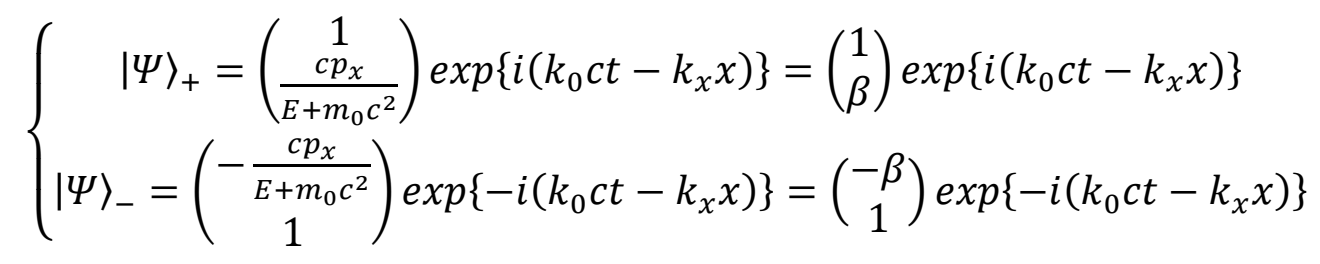

In Eq. (4), $\beta$ is the relativistic factor $(u / c),|\Psi\rangle_{+}$and $|\Psi\rangle_{-}$are respectively the particle and antiparticle spinors. Wave vectors $k_{0}$ and $k_{x}$ are:

$\left\{\begin{array}{l}k_{0}=\frac{p_{0}}{\hbar}=\frac{\gamma m_{0} c}{\hbar} \\ k_{x}=\frac{p_{x}}{\hbar}=\frac{\gamma m_{0} u}{\hbar}\end{array}\right.$

where $\gamma$ is the Lorentz factor. At the beginning of the barrier $\left(x=x_{0}\right)$ the De Broglie wave may be reflected or transmitted. In the latter case, the wave behaves like an evanescent stationary wave [13], which the Schrödinger equation describes. It has the wave vector:

$$
\left\{\begin{array}{cc}
\chi_{+}=\frac{\sqrt{2 m_{0}(U-E)}}{\hbar} & \text { Particle } \\
\chi_{-}=\frac{\sqrt{2 m_{0}(U+E)}}{\hbar} & \text { Antiparticle }
\end{array}\right.
$$

At the end of the barrier, the evanescent wave is transmitted in region III, where it returns to be a free relativistic particle with half-integer spin. Since the wave function (4) is a two-component vector, we must study the scattering process for both of them. The peculiarity of this model is that inside the barrier, the particle/antiparticle no longer has a relativistic behaviour. At the barrier edges, the Dirac spinor and the Schrödinger evanescent wave have to match the continuity conditions that quantum theory imposes.

We will investigate the tunneling time by the phase time defined as [11]:

$\tau=\hbar \frac{\partial \arg \left\{C_{T} \exp \left[i\left(k_{0} c t_{a}-k_{x} x_{a}\right)\right]\right\}}{\partial E}$ 
Therefore, the study object of this research preliminarily requires the calculation of the transmission amplitude $C_{T}$.

\section{$4 \quad$ Transmission and Reflection Amplitudes}

Let us calculate the transmission and reflection amplitudes of the scattering process that we will use for tunneling time investigation. Considering initially the first component of the particle spinor (4), the continuity conditions are:

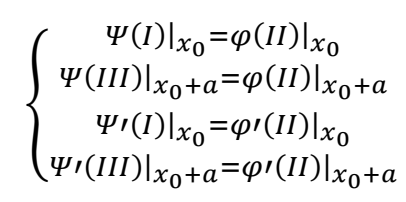

where:

$$
\left\{\begin{array}{c}
\Psi(I)=\exp \left\{i\left(k_{0} c t-k_{x} x\right)\right\}+c_{R} \exp \left\{-i\left(k_{0} c t-k_{x} x\right)\right\} \\
\varphi(I I)=\alpha e^{\chi x}+\delta e^{-\chi x} \\
\Psi(I I I)=c_{T} \exp \left\{i\left(k_{0} c t-k_{x} x\right)\right\}
\end{array}\right.
$$

$c_{R}$ and $c_{T}$ are respectively the reflection and the transmission coefficients of the particle wave, while $\alpha$ and $\delta$ are the coefficients of the evanescent wave (see Appendix A). Substituting functions (9) in (8) and solving the system by the Cramer algorithm, we get:

$$
c_{T}=\frac{4 i \chi \Delta k}{\exp \left\{i\left(k_{0} c t_{a}-k_{\chi} x_{a}\right)\right\}\left[e^{\chi x} a(\chi-i \Delta k)^{2}+e^{-\chi x} a(\Delta k-i \chi)^{2}\right]}
$$

$C_{R}=\frac{\exp \left\{2 i\left(k_{0} c t_{0}-k_{x} x_{0}\right)\right\} \exp \left\{i\left(k_{0} c t_{a}-k_{x} x_{a}\right)\right\}}{4\left(\chi^{2}+\Delta k^{2}\right) \sinh ^{2}\left(\chi x_{a}\right)}\left[-2 \sinh \left(\chi x_{a}\right)\left(\chi^{2}-\Delta k^{2}\right)-4 i \exp \left\{i\left(k_{0} c t_{0}-\right.\right.\right.$

$\left.\left.\left.k_{x} x_{0}\right)\right\} \exp \left\{i\left(k_{0} c t_{a}-k_{x} x_{a}\right)\right\} \cosh \left(\chi x_{a}\right)\right]$

where $\Delta k=\left(k_{0}-k_{1}\right)=\gamma m_{0}(c-u) / \hbar$. For antiparticle states, Eq. (10) provides the transmission amplitude, replacing $\Delta k$ with $-\Delta k$ and using the Schrödinger wave vector $\chi_{-}$. Through some algebraic manipulations, we use the explicit forms (5) and (6) of the Dirac and Schrödinger wave vectors and set $m_{0} \equiv c \equiv \hbar=1$. Eq. (10) thus provides the probability transmission of the particle:

$C_{T}^{2}=\frac{\gamma^{2}(1-u)^{2}(U-E)}{\left[\gamma^{2}(1-u)^{2}+2(U-E)\right] \sinh ^{2}\left(x_{a} \sqrt{2(U-E)}\right)}$

When increasing the barrier height, i.e., increasing the potential energy $U$, the probability that the particle is transmitted through the barrier rapidly goes to zero, while if $U=E$ this probability tends to one:

$\left\{\begin{array}{l}\lim _{U \rightarrow \infty} C_{T}^{2}=0 \\ \lim _{U \rightarrow E} C_{T}^{2}=1\end{array}\right.$

The limits (13) hold also for the antiparticle state, but for $U \rightarrow \infty$ the probability tends to zero more rapidly. The reflection probability $C_{R}^{2}$ has an opposite trend to the transmitted one, which the quantum relation $\left(C_{T}^{2}+C_{R}^{2}\right)=1$ has to satisfy. 


\section{$5 \quad$ Tunneling Time for Particle States}

As mentioned earlier, the phase time (7) helps perform the investigation of the tunneling time. The first step for its calculation is to determine the argument of the complex number $C_{T} \exp \left[i\left(k_{0} c t_{a}-k_{x} x_{a}\right)\right]$. To simplify the formalism, from now on the Greek letter $\theta$ will represent this. Through some algebraic calculations (see Appendix B), we get:

$$
\theta=\operatorname{arctg}\left[-2 \chi \Delta k \frac{1}{\Delta k^{2}+\chi^{2} \frac{e^{2 \chi x} a-1}{e^{2 \chi x} a+1}}\right]
$$

Assuming that the barrier width is large enough, we may set the term $\left(e^{2 \chi x_{a}}-1 / e^{2 \chi x_{a}}+1\right)$ equal to one, so that the argument function becomes:

$\theta=\operatorname{arctg}\left[-\frac{2 \chi \Delta k}{\Delta k^{2}+\chi^{2}}\right]$

Performing its derivative respect for the particle energy and substituting the result in Eq. (7), we get the phase time (see Appendix C):

$\tau^{\left(1^{\circ}\right)}=\frac{2 m_{0} \Delta k E\left(1-2 \chi^{2}\right)+2 \hbar^{2} \chi^{2} \Delta k\left(2 \Delta k^{2}-1\right)}{\hbar \chi E\left[\left(\Delta k^{2}+\chi^{2}\right)^{2}+4 \Delta k^{2} \chi^{2}\right]}$

where the superscript $\left(1^{\circ}\right)$ means that the phase time refers the first component of the Dirac spinor. Eq. (16) is independent from the barrier width $a$ and, as expected, the Hartman effect [11] occurs. Moreover, the value of the phase time is always negative whatever the particle energy and the barrier height. According to references [22-23], this means the tunneling always occurs at superluminal velocities. Figure 2 shows the phase time trends for $U=10$ and $U=100$ vs the relativistic factor $\beta$ (as usual we set $m_{0} \equiv c \equiv \hbar=1$ ):

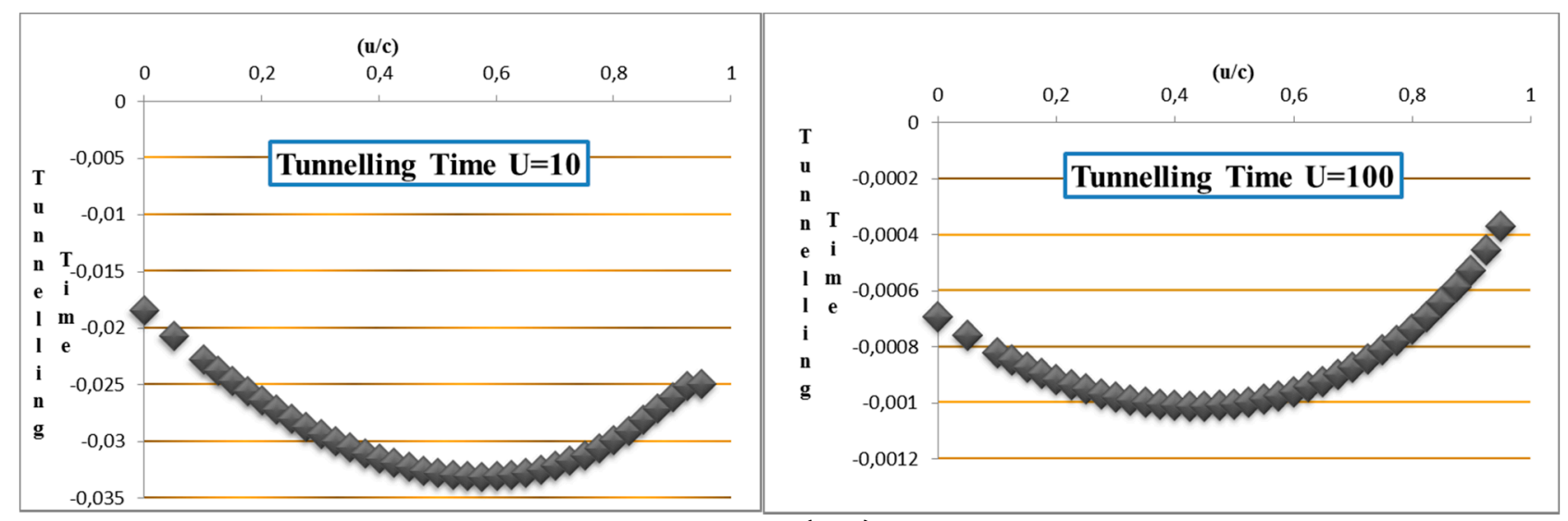

Figure 2: Tunneling time vs relativistic factor $\beta=(u / c)$ for two potential energies. All values are negative, proving that the tunneling is always superluminal. As the barrier height increases, the tunneling time tends to zero.

The trends show a minimum tunneling time that shifts towards low values of the relativistic factor as the barrier height increases. Moreover, when increasing the barrier height, the phase time goes rapidly to zero. This result is in agreement with the superluminality that the Heisenberg uncertainty principle predicts. The ratio between the barrier width and the tunneling time provides the barrier-crossing speed, at least in principle. From the obtained results, we thus conclude that the higher the barrier is, the faster the scattering through it is. This phenomenon occurs as if the potential barrier boosts the particle towards higher velocities, even if its occurrence probability (that the squared transmission amplitude provides) goes to zero as the barrier height decreases. 
We may also display the superluminal tunneling by the refraction index, calculated as the ratio between the velocity of the free particle before impinging on the barrier and its velocity during the tunneling:

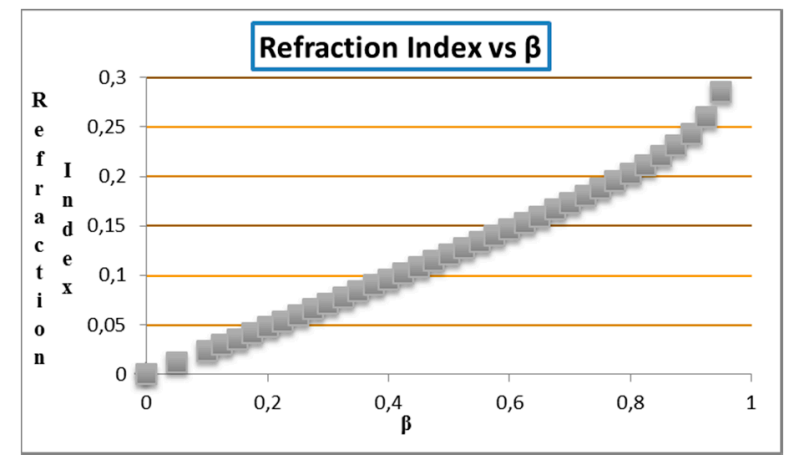

Figure 3: Tunneling refraction index vs relativistic factor $\beta=(u / c)$ for the barrier height $U=10$. All values are lower than one, proving that the tunneling is always superluminal.

As expected, the refraction index is always lower than one and increases with the relativistic factor. This means that as the velocity of the free particle approaches the speed of light, the superluminality of the tunneling tends to decrease, and the particle velocity inside the barrier becomes luminal. This result is explainable by the fact that by keeping the potential barrier constant and increasing the particle velocity, the energy gap rapidly tends to zero.

Let us calculate the phase time for the second component of the spinor (4) for the particle state following the same physico-mathematical approach used for the first component (see Appendix D):

$$
\tau^{\left(2^{\circ}\right)}=\hbar \frac{2\left(1-\beta^{2}\right)\left(\Delta k^{2}+\chi^{2}\right)}{E\left[\left(\Delta k^{2}+\chi^{2}\right)^{2}+4 \beta^{4} \Delta k^{2} \chi^{2}\right]}+\tau^{\left(1^{\circ}\right)}
$$

This shows that the two components of the spinor emerge from the far side of the barrier with a phase time difference given by:

$\Delta \tau=\hbar \frac{2\left(1-\beta^{2}\right)\left(\Delta k^{2}+\chi^{2}\right)}{E\left[\left(\Delta k^{2}+\chi^{2}\right)^{2}+4 \beta^{4} \Delta k^{2} \chi^{2}\right]}$

This quantity, which from now we will call phase time delay, is always positive. Figure 4 shows its trend:

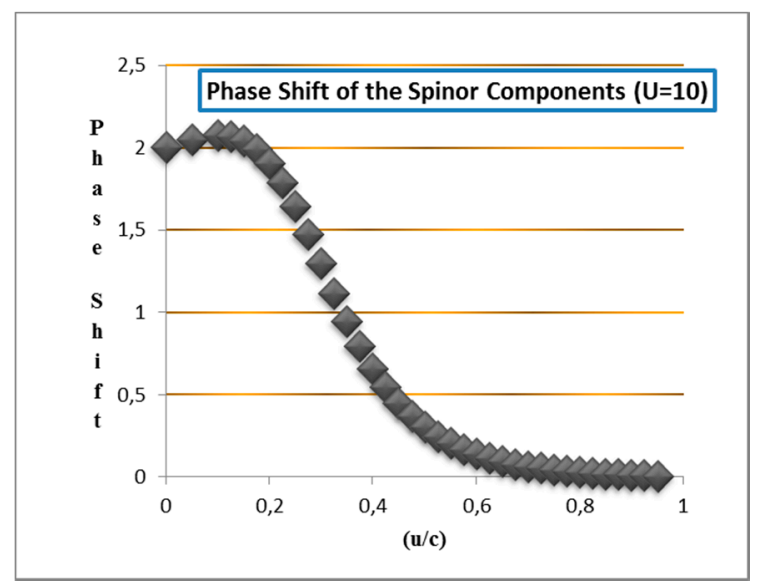

Figure 4: Phase time difference of the two spinor components for the barrier height $U=10$. The quantum tunneling effect twists the spinor, and the deformation disappears when increasing the free particle velocity. 
This delay is considerable at non-relativistic regimes $(u \ll c)$, at which the magnitude of the secondary component of spinor (4) is very low, and tends to zero when the particle velocity approaches the speed of light. Overall, the phase time (17) is positive and follows a trend very similar to that of Figure 4. For this reason, whatever the barrier width, the secondary spinor component always emerges later than the principal one. This phenomenon leads to an anomalous distortion of the Dirac wave function that disappears when the relativistic behaviour of the particle becomes relevant.

\section{Tunneling Time for Antiparticle States}

Let us calculate the tunneling time for the antiparticle states following the same approach used in the previous section. The negative values of energy $E$ and the relativistic wave number $\Delta k$ represent the only difference. Eq. (16) can then provide the phase time by replacing $E$ with $-E, \Delta k$ with $-\Delta k$ and using the second variant of Eq. (6):

$$
\tau_{-}^{\left(1^{\circ}\right)}=-\frac{2 m_{0} \Delta k E\left(2 \chi^{2}-1\right)+2 \hbar^{2} \chi^{2} \Delta k\left(1-2 \Delta k^{2}\right)}{\hbar \chi E\left[\left(\Delta k^{2}+\chi^{2}\right)^{2}+4 \Delta k^{2} \chi^{2}\right]}
$$

The negative sign of $\Delta k$ is due to the fact that the sign of the argument of the exponential function for the antiparticle state is opposite to that of the particle state. Eq. (19) does not depend on the barrier width, and the Hartman effect occurs. Figure 5 shows the trend for $U=10$ :

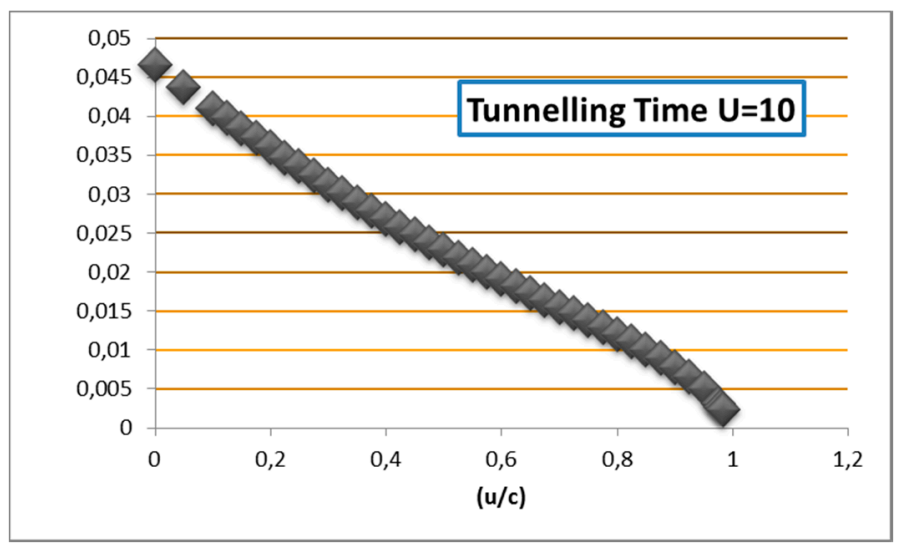

Figure 5: Phase time vs relativistic factor $\beta$ for the antiparticle state for the barrier height $U=10$. The phase time is always positive and tends to zero as the antiparticle velocity approaches the speed of light.

Unexpectedly the phase time is always positive, and the tunneling through the barrier could be subluminal or superluminal depending on the barrier width. In particular, the higher the barrier width is, the higher the tunneling velocity is. Moreover, when increasing the barrier height, the phase time gets smaller and smaller. These behaviours are quite similar to those obtained for the particle state. Finally, once setting the barrier height, the antiparticle phase time tends to zero as the velocity approaches the speed of light, just as what occurs for the particle state.

Similarly, Eq. (18) provides the phase time difference between the two components of the antiparticle spinor, replacing $E$ with $-E$ and using the second part of Eq. (6). Figure 6 shows its trend: 


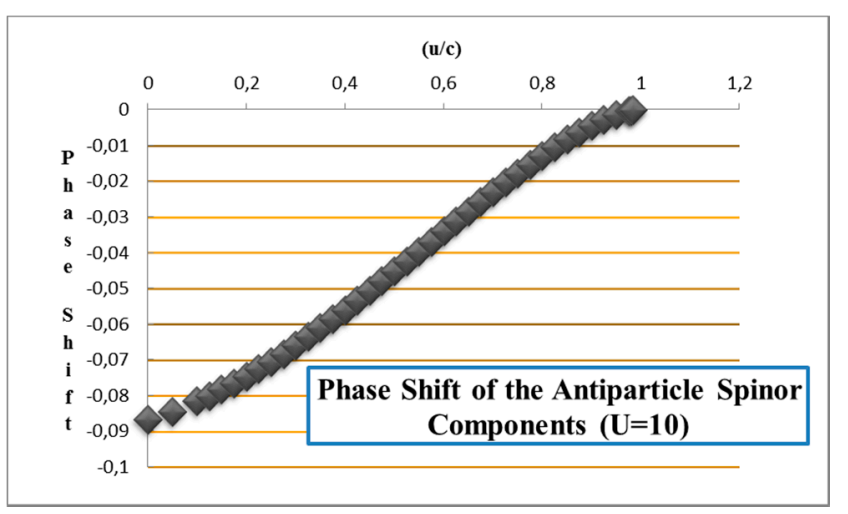

Figure 6: Phase time difference of the two antiparticle spinor components for the barrier height $U=10$. The quantum tunneling effect twists the spinor, and the deformation disappears as the free particle velocity increases.

Once again unexpectedly, the secondary component of the antiparticle spinor emerges from the far side of the barrier before the principal one. This always leads to an anomalous distortion of the Dirac antiparticle spinor that, however, is opposite to the one that occurs for the particle state. This distortion disappears as the antiparticle velocity approaches the speed of light but is slower than what occurs for the particle spinor.

\section{Discussion}

This study proves that relativistic free particles and antiparticles behave differently when a potential barrier scatters them, even at velocities close to the speed of light. For large barrier widths, these behaviours tend to converge and become uniform. Although the Hartman effect occurs for both particles and antiparticles, the tunneling is always superluminal for particle states. However, it can be subluminal or superluminal for antiparticle states depending on the barrier width. The interaction of the relativistic wave packet with the potential barrier generates superluminality. Inside this, forward and backward evanescent waves describe the particle or antiparticle. As references $[13,24]$ suggest, this interaction leads to a complex set of interferences between incident and reflected waves that cause an acceleration of the incoming wave. However, the Heisenberg uncertainty principle may also explain superluminal tunnelling. The energy confinement of the particle within the barrier reduces the time uncertainty, and consequently increases the one affecting the energy. Such high uncertainty leads to the transmitted wave exceeding the speed of light. We think this interpretation of the phenomenon we are investigating is more understandable and closer to quantum theory.

We must still explain the reason why an antiparticle behaves differently than a particle, i.e., why it does not always tunnel in superluminal mode. The negative energy of the antiparticle decreases the transmission probability that Eq. (12) provides. This is especially due to the strong increases of the hyperbolic function at the denominator and changes the interference mechanism between forward and backward waves. In other words, the barrier tends to reflect an antiparticle more than a particle. An antiparticle tunneling through the potential barrier $U$ is equivalent to a particle that tunnels through a potential barrier $(U+E)$. This provides a more physical explanation of why an antiparticle has a lower probability of penetrating the barrier. However, once the antiparticle overcomes the barrier edge, it becomes more energy confined than the particle and accelerates more strongly. In fact, comparing the first trend of Figure $\mathbf{2}$ with that of Figure $\mathbf{5}$ shows that as the particle/antiparticle velocity approaches the speed of light, the phase time of the antiparticle tends to zero faster than what occurs for the particle.

If the high-energy confinement (very high barriers) facilitates acceleration towards superluminal velocity, why does increasing $\beta$ tend to decrease the phase time? Increasing the relativistic factor decreases the energy gap between the barrier and the particle/antiparticle. This reduces the energy confinement and, consequently, the uncertainty affecting the energy $E$. But because $E$ is so high, it is small enough of an uncertainty to boost the particle towards ever higher 
superluminal velocities. In fact, the trends show that accelerating the particle/antiparticle within the barrier is a more important kinetic factor than the energy confinement.

Finally, the different mechanism of interference between the transmitted and reflected waves may also explain spinor distortion. This provides different accelerations for the principal and secondary spinor components [24]. We can also state that the potential barrier contributes to spread the wave packet. An earlier occurrence or delay of the secondary spinor component is equivalent to frequency changes, i.e., energy changes between the plane waves forming the wave packet.

\section{Conclusion}

To summarize, we have considered tunneling of a relativistic free particle with half-integer spin through a one-dimensional potential barrier. The analysis suggests that the total transmission is always superluminal for particle states and may be subluminal or superluminal for antiparticle states. Within the potential barrier the two components of the spinor behave as evanescent waves and the high energy confinement accelerates the particle/antiparticle further the speed of light. The phenomenon may be explained considering the interference between forward and backward evanescent waves, but a more easily explanation holds if the Heisenberg uncertainty principle is taken in account. As the particle/antiparticle velocity approaches the speed of light the tunneling time tends to zero making diverge the acceleration toward infinite values. The study also proves that the two spinor transmitted components emerge from the potential barrier at different times, providing an anomalous distortion of the Dirac wavefunction. This distortion occurs in an opposite way for particle and antiparticle. More precisely, the secondary spinor component is delayed compered the first one for particle states and is anticipated for antiparticle states. All these results are innovative with respect to those available in the literature and suggest a way to obtain a source of superluminal massive elementary particles: ultra-relativistic particles scattering through huge potential barriers. As discussed above, the probability of total transmission of the particle/antiparticle through the barrier strongly decreases as its incoming velocity approaches the speed of light, but the occurrence of the model used in this study cannot be excluded a priori in a cosmological scenario.

\section{References}

1. Steinberg, A.M.; Kwiat, P.G.; Chiao, R.Y.; Measurement of the Single-Photon Tunneling Time 1993, Phys. Rev. Lett., 71, 708-711. DOI: 10.1103/PhysRevLett.71.708.

2. Spielmann, C.; Szipöcs, R.; Stingl, A.; Krausz, F.; Tunneling of Optical Pulses Through Photonic Band Gaps 1994, Phys. Rev. Lett., 73, 2308-2312. DOI: 10.1103/PhysRevLett.73.2308.

3. Recami, E.; Superluminal Waves and Objects: an Overview of the Relevant Experiments 2009, J. of Phys: Conference Series, 196, 012020. DOI: 10.1088/1742-6596/196/1/012020.

4. Ehrlich, R.; Tachyonic Neutrinos and the Neutrinos Masses 2013, Astropart. Phys., 41, 1-6. DOI: 10.1016/j.astropartphys.2012.09.009.

5. Pradhan, R.K.; Singh, L.P.; On Reality of Tachyon de Broglie Waves 2009, arXiv: 0908.1365 [Physics.gen-ph].

6. Mirabel, I.F.; Rodríguez, L.F.; A Superluminal Source in the Galaxy 1994, Nature, 371, 46-48. DOI: 10.1038/371046a0.

7. Landauer, R.; Martin, T.; Barrier Interaction Time in Tunneling 1994, Rev. Modern Phys., 66, $217-228$. DOI: 10.1103/RevModPhys.66.217.

8. Davies, P.C.W.; Quantum Tunneling Time 2005, Am. J. Phys., 73, 23-27. DOI: 10.1119/1.1810153.

9. Chuprikov, N.; New Approach to Quantum Tunneling Process: Characteristic Times for Transmission and Reflection 2006, Russian Phys. J., 49(3), 314-325. DOI: 10.1007/s11182-006-0106-9.

10. Hauge, E.H.; Stovneng, J.A.; Tunneling Times: a Critical Review 1989, Rev. Mod. Phys., 61, 917-936.

11. Hartman, T.E.; Tunneling of a Wave Packet 1962, J. Appl. Phys., 33(12), 3427-3433. DOI: 10.1063/1.1702424.

12. Winful, H.G.; Tunneling Time, the Hartman Effect and Superluminality: a Proposed Resolution of an Old Paradox 2011, Phys. Rep., 436(1-2), 1-69. DOI: 10.1016/j.physrep.2006.09.002.

13. Recami, E.; Superluminal Tunneling Through Successive Barriers: Does Quantum Mechanics Predict Infinite Group Velocities? 2004, J. Mod. Optics, 51(6-7), 913-923. DOI: 10.1080/09500340410001664386. 
14. Petrillo, V.; Janner, D.; Relativistic Analysis of a Wave Packet Interacting with a Quantum-Mechanical Barrier 2003, Phys. Rev. A, 67, 012110. DOI: 10.1103/PhysRevA.67.012110.

15. Chuprikov, N.; Tunneling Time Problem: at the Intersection of Quantum Mechanics, Classical Probability Theory and Special Relativity 2014, arXiv: 1303.6181 [Quantum-ph].

16. Muga, G.; Ruschhaupt, A.; del Campo, A. (eds.); Time in Quantum Mechanics, Vol. 2 2009, Springer, Berlin.

17. Bandopadhyay, S.; Jayannavar, A.M.; Phase Time for a Tunneling Particle 2005, arXiv: 0511089 [Quantum-ph].

18. Wigner, E.P.; Lower Limit for the Energy Derivative of the Scattering Phase Shift 1955, Phys. Rev. B, 98, 145-147. DOI: 10.1103/PhysRev.98.145.

19. Merzbacher, E.; The Early History of Quantum Tunneling 2002, Phys. Today, 55(8), 44-49.

20. Nanni. L.; Quantum Theory of Half-Integer Spin Free Particles from the Perspective of the Majorana Equation 2016, arXiv: 1603.05965 [Physics.gen-ph].

21. Park, S.T.; Propagation of a Relativistic Electron Wave Packet in the Dirac Equation 2012, Phys. Rev. A, 86, 1-12. DOI: 10.1103/PhysRevA.86.062105.

22. Rosas-Ortiz, O.; Cruz, S.C.; Fernández-García, N.; Negative Time Delay for Wave Reflection from a OneDimensional Semi-Harmonic Well 2012, arXiv: 1208.4317 [Quant-ph].

23. Bernardini, A.E.; Relativistic Tunneling and Accelerated Transmission 2008, arXiv: 0706.3930 [Quant-ph].

24. Lee, Y.J.; Park, J.C.; Superluminal Dirac Wave-Packet Tunneling Through a Wide Potential Barrier of Low Energy 2004, J. Korean Phys. Soc., 44(4), 804-808. 


\section{Appendix A}

The Cramer rule can solve linear system (8). To simplify the mathematical formalism, we set:

$$
\left\{\begin{array}{c}
l_{1}=\exp \left\{i\left(k_{0} c t_{0}-k_{x} x_{0}\right)\right\} \\
l_{2}=\exp \left\{-i\left(k_{0} c t_{0}-k_{x} x_{0}\right)\right\} \\
m_{1}=e^{\chi x_{0}} \\
m_{2}=e^{-\chi x_{0}} \\
n_{1}=e^{\chi x_{a}} \\
n_{2}=e^{-\chi x_{a}} \\
q_{1}=\exp \left\{i\left(k_{0} c t_{a}-k_{x} a_{0}\right)\right\}
\end{array}\right.
$$

The determinant of the coefficient matrix $A$ is:

$$
\operatorname{det}(A)=l_{2} m_{2} n_{1} q_{1}(\chi-i \Delta k)^{2}+l_{2} m_{1} n_{2} q_{1}(i \Delta k-\chi)^{2}
$$

The four unknown quantities, i.e., the coefficients of the linear combination are:

$$
\left\{\begin{array}{c}
C_{R}=\frac{l_{1} q_{1}}{\operatorname{det}(A)}\left[\left(n_{1} m_{2}-m_{1} n_{2}\right)\left(\chi^{2}-\Delta k^{2}\right)-21 q_{1} \Delta k \chi\left(m_{1} n_{2}+m_{2} n_{1}\right)\right] \\
C_{T}=-4 i \frac{l_{1} l_{2} n_{1} n_{2} \Delta k \chi}{\operatorname{det}(A)} \\
\alpha=2 \frac{\left[l_{1} l_{2} n_{2} q_{1} \Delta k(\Delta k-i \chi)\right]}{\operatorname{det}(A)} \\
\delta=-2 \frac{\left[l_{1} l_{2} n_{1} q_{1} \Delta k(\Delta k+i \chi)\right]}{\operatorname{det}(A)}
\end{array}\right.
$$

\section{Appendix B}

The calculation of phase time requires knowledge of the complex number argument:

$$
z=C_{T} \exp \left[i\left(k_{0} c t_{a}-k_{x} x_{a}\right)\right]
$$

Using (A1) and considering that $(\chi-i \Delta k)^{2} / i=(i \Delta k-\chi)^{2}$ and $(\Delta k-i \chi)^{2} / i=(i \chi-\Delta k)^{2}$, we get:

$$
z=-\chi \Delta k \frac{1}{e^{\chi x a} a(i \Delta k-\chi)^{2}+e^{-\chi x} a(i \chi-\Delta k)^{2}}
$$

Solving the squares of the two binomials in (B2), we obtain:

$$
z=-\chi \Delta k \frac{1}{\Delta k^{2}\left(e^{\chi x} a+e^{-\chi x} a\right)+\chi^{2}\left(e^{\chi x} a-e^{-\chi x} a\right)-2 i \chi \Delta k\left(e^{\chi x} a+e^{-\chi x} a\right)}
$$

Denoting by $z^{\prime}$ the denominator of (B3) and recalling that its inverse is $z^{\prime-1}=\overline{z^{\prime}} /\left|z^{\prime}\right|^{2}$ and that $\operatorname{arctg}(\beta / \alpha)$ provides the argument of a complex number $z=\alpha+i \beta$, we obtain:

$$
\arg (z)=\operatorname{arctg}\left[-2 \chi \Delta k \frac{\left(e^{\chi x_{a}}+e^{-\chi x_{a}}\right)}{\Delta k^{2}\left(e^{\chi x} a+e^{-\chi x_{a}}\right)+\chi^{2}\left(e^{\chi x} a-e^{-\chi x_{a}}\right)}\right]
$$

Some simple algebraic manipulations allow us to rewrite (B4) as:

$$
\arg (z)=\operatorname{arctg}\left[-2 \chi \Delta k \frac{1}{\Delta k^{2}+\chi^{2} \frac{e^{2 \chi x} a-1}{e^{2 \chi x} a+1}}\right]
$$


If the barrier width $a$ is large enough, then the fraction $\frac{e^{2 \chi x} a-1}{e^{2 \chi x} a+1} \cong 1$, and (B5) becomes:

$$
\arg (z)=\operatorname{arctg}\left[-2 \frac{\chi \Delta k}{\Delta k^{2}+\chi^{2}}\right]
$$

which is independent of the barrier width.

\section{Appendix C}

In this section we explain the steps to get the derivative of the argument function (B6). Using the chain rule shows:

$$
\frac{\partial \arg (z)}{\partial E}=\frac{1}{1+\frac{4 \Delta k^{2} \chi^{2}}{\left(\Delta k^{2}+\chi^{2}\right)^{2}}} \frac{\partial}{\partial E}\left(-2 \frac{\chi \Delta k}{\Delta k^{2}+\chi^{2}}\right)=\frac{\left(\Delta k^{2}+\chi^{2}\right)^{2}}{\left(\Delta k^{2}+\chi^{2}\right)^{2}+4 \Delta k^{2} \chi^{2}} \frac{\partial}{\partial E}\left(-2 \frac{\chi \Delta k}{\Delta k^{2}+\chi^{2}}\right)
$$

The last derivative of (C1) is:

$$
\frac{\partial}{\partial E}\left(-2 \frac{\chi \Delta k}{\Delta k^{2}+\chi^{2}}\right)=\frac{-2\left(\chi^{\prime} \Delta k+\chi \Delta k^{\prime}\right)\left(\Delta k^{2}+\chi^{2}\right)+2 \chi \Delta k\left(2 \Delta k \Delta k^{\prime}+2 \chi \chi^{\prime}\right)}{\left(\Delta k^{2}+\chi^{2}\right)^{2}}
$$

Now we calculate the derivatives $\chi^{\prime}$ and $\Delta k^{\prime}$ separately using the first parts of Eq. (5) and (6) and by performing some simple algebraic manipulations:

$$
\left\{\begin{array}{c}
\chi^{\prime}=\frac{\partial}{\partial E} \frac{\sqrt{2 m_{0}(U-E)}}{\hbar}=-\frac{m_{0}}{\hbar^{2} \chi} \\
\Delta k^{\prime}=\frac{\partial}{\partial E} \frac{\gamma m_{0}}{\hbar}(c-u)=\frac{\partial}{\partial E} \frac{E}{c \hbar} \frac{1-u}{1-c}=\frac{\Delta k}{E}
\end{array}\right.
$$

Substituting the results (C3) into derivative (C2) and then the final expression into (C1), we get:

$$
\frac{\partial \arg (z)}{\partial E}=\frac{2 m_{0} \Delta k E\left(1-2 \chi^{2}\right)+2 \hbar^{2} \chi^{2} \Delta k\left(2 \Delta k^{2}-1\right)}{\hbar^{2} \chi E\left[\left(\Delta k^{2}+\chi^{2}\right)^{2}+4 \Delta k^{2} \chi^{2}\right]}
$$

from which we obtain the particle phase time (16).

\section{Appendix D}

The argument function of the secondary component of spinor (4) is:

$$
\arg \left(z^{2^{\circ}}\right)=\operatorname{arctg}\left[-2 \beta^{2} \frac{\chi \Delta k}{\Delta k^{2}+\chi^{2}}\right]
$$

Before calculating its derivative, we have to explain the relativistic factor $\beta$ as the function of the particle energy. We proceed as follows:

$$
E=\frac{1}{\sqrt{1-\beta^{2}}} m_{0} c^{2} \Rightarrow m_{0}^{2} c^{4}=E^{2}\left(1-\beta^{2}\right) \Rightarrow \beta^{2}=\frac{E^{2}-m_{0}^{2} c^{4}}{E^{2}}
$$

Now we calculate the derivative of function (D1) using the chain rule: 


$$
\begin{aligned}
& \frac{\partial \arg \left(z^{2^{\circ}}\right)}{\partial E}=\frac{\left(\Delta k^{2}+\chi^{2}\right)^{2}}{\left(\Delta k^{2}+\chi^{2}\right)^{2}+4 \beta^{4} \Delta k^{2} \chi^{2}} \frac{\partial}{\partial E}\left(-2 \beta^{2} \frac{\chi \Delta k}{\Delta k^{2}+\chi^{2}}\right)= \\
& =\frac{\left(\Delta k^{2}+\chi^{2}\right)^{2}}{\left(\Delta k^{2}+\chi^{2}\right)^{2}+4 \beta^{4} \Delta k^{2} \chi^{2}}\left[\frac{\partial}{\partial E}\left(-2 \frac{\chi \Delta k}{\Delta k^{2}+\chi^{2}}\right)+\frac{\partial}{\partial E}\left(\beta^{2}\right)\right]
\end{aligned}
$$

We have already calculated the first derivative in the square brackets in Appendix $\mathbf{C}$. We calculate the second derivative using expression (D2):

$$
\frac{\partial}{\partial E}\left(\beta^{2}\right)=\frac{\partial}{\partial E} \frac{E^{2}-m_{0}^{2} c^{4}}{E^{2}}=2 \frac{\left(1-\beta^{2}\right)}{E}
$$

Substituting the results (D4) and (C2) into (D3), we get:

$$
\frac{\partial \arg \left(z^{2^{\circ}}\right)}{\partial E}=2 \frac{\left(\Delta k^{2}+\chi^{2}\right)^{2}}{\left(\Delta k^{2}+\chi^{2}\right)^{2}+4 \beta^{4} \Delta k^{2} \chi^{2}} \frac{\left(1-\beta^{2}\right)}{E}+\frac{\partial \arg (z)}{\partial E}
$$

from which we obtain the phase time (17) of the secondary spinor component. 